\title{
Fragen an Homers Achilleus ${ }^{1}$
}

\author{
Joachim Latacz
}

Universität Basel, Departement Altertumswissenschaften

Homer-Kommentar, Petersgraben 51,CH-4051 Basel, Schweiz; joachim.latacz@unibas.ch

For citation: Joachim Latacz. Fragen an Homers Achilleus. Philologia Classica 2018, 13(1), 9-25. https://doi. org/10.21638/11701/spbu20.2018.101

To counter the predominance of narratology and other self-serving approaches to the Ilias of Homer which have swarmed the recent criticism, the author of this article poses a number of simple questions to Achilles himself eliciting answers through the clues the text of the Ilias alone can offer. "What is your role in the Ilias?" "Pivotal". "What caused your wrath and withdrawal from battle?" "Self-respect and abhorrence of the ostentatiously lavish gifts meant to insult". "Why then did you return to battle in the end?" "The death of Patrocles has dealt such a blow that there is no longer any heeding of trifles, the wrath giving way to vengeance which exhausts itself, but only by way of compassion." "Is there anything you value most?" "It is but life itself, the loss of which is irrecoverable, and not, as one could over-hastily conclude, undying glory." With the help of these questions, the author hopes to tap into the very heart of this epic poem and its main hero, Achilles, making it evident each time that Homer masterfully goes beyond the boundaries of what is traditionally believed to be the heroic code of conduct thus making it richer and less clear-cut. The voice of Achilles reaching to us through millennia is a living voice. Keywords: Achilles, Ilias, Homer, Greek epic poetry.

Dem Betrachter der neuesten Homer-Literatur drängt sich der Eindruck auf, dass es heute immer öfter um 'schicke' Themenformulierungen geht, wie etwa: 'Die strukturelle Strategie Homers im Spannungsfeld von Narratologie und moderner Performance-Theorie. Dem Thema entspricht dann meist der Inhalt: Mehr Kunstwerk der Interpretation als

${ }^{1}$ Festvortrag beim Empfang des Ausonius-Preises der Universität Trier 2014. - Auf Wunsch des Herausgebers wurde der Vortragsstil weitgehend beibehalten (die ungewohnt häufigen Kursivierungen etc. versuchen etwas Ersatz für die fehlende viva vox zu schaffen). - Eine umfassende rezeptionsgeschichtliche Behandlung des Themas von der Gegenwart bis zurück zu Homer bietet Verf. in: J. Latacz, Achilleus. Wandlungen eines europäischen Heldenbildes, Leipzig, Teubner, $1995 ;{ }^{2} 1997,108$ S. (wieder abgedruckt in: J. Latacz, Homers Ilias. Studien zu Dichter, Werk und Rezeption. Hrsg. v. Thierry Greub, Krystyna Greub-Frącz und Arbogast Schmitt. Berlin — Boston, Walter de Gruyter, 2014 (BzA 327), 267-346).

(C) St. Petersburg State University, 2018 
Interpretation des Kunstwerks. - Nichts davon hier! Statt dessen in naiver Schlichtheit nur ein paar Fragen - Fragen aber, die vielleicht zu einem tieferen Verständnis von Qualität und Sinngehalt der Ilias führen könnten.

Adressat der Fragen soll allerdings nicht das Kunstwerk selbst direkt sein - das reagiert ja doch nur - siehe Platons Phaidros! (Phdr. $275 \mathrm{~d}$ ) - mit erhabenem Schweigen. Adressat soll vielmehr ein besonders kompetenter Helfer für das Verständnis dieses Kunstwerks sein: Achilleus.

Es sind ganz einfache Fragen, die wir an Homers Achilleus richten wollen:

1. Welche Rolle spielst Du in Ilias und Odyssee?

2. Was ist die eigentliche Ursache für Deinen Groll ( $\mu$ ๆ̃vı) und für Deinen Kampfboykott?

3. Warum lehnst Du das Wiedergutmachungsangebot Agamemnons (im 9. IliasGesang) ab?

4. Was ist für Dich der höchste Wert?

$\mathrm{Zu}$ Frage 1: Worauf wird Achilleus in seiner Antwort wohl zuerst verweisen? Sicherlich auf das Prooimion der Ilias. „Das wird nicht immer richtig verstanden“, wird er wahrscheinlich sagen. Sehen wir uns also dieses Prooimion, das jeder bestens zu kennen glaubt, erneut an:

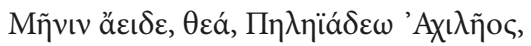

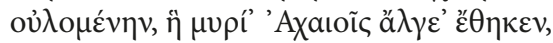

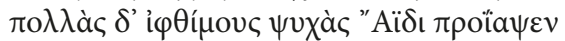

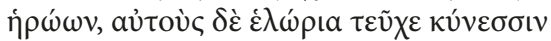

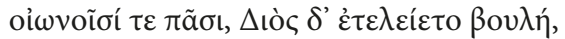

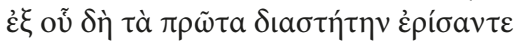

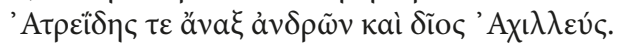

„Den Groll singe, Göttin, des Pēlēïaden Achilleus, den ganz unsel'gen! der zahllose Schmerzen den Achaiern brachte und viele starke Leben dem Gott Hades zuwarf — Leben von Helden - und sie selbst zum Fraße werden ließ für Hunde, und für die Vögel zum Bankett (Zeus' Wille war's, der sich darin erfüllte!), von dem Moment an, da zerstritten auseinandertraten der Atreïde, Herr der Männer, und der göttliche Achilleus.“

(Übers.: J.L.)

Wir sehen: Die Thema-Angabe der Ilias, die der erste Verse enthält, ist sehr kunstvoll aufgebaut: Fünf Wörter - zwei am Vers-Anfang, zwei am Vers-Ende - in der Mitte getrennt durch das fünfte: die Adressatin des anrufenden Sängers: die Göttin, die Muse, $\theta \varepsilon \dot{\alpha}$. Lassen wir die drei mittleren Wörter weg, dann ergibt sich das reine Thema. Es rahmt den

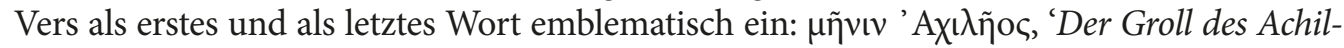
leus'.

Das also ist das Thema der Ilias. Nicht Troia und nicht der Troianische Krieg. Darum geht es nicht. Worum es wirklich in der Ilias geht, das ist im letzten Kern - Achilleus. Die 
meisten Übersetzungen in moderne Sprachen lassen das unkenntlich werden. Das fängt bereits mit J.H. Voß an (1793):

Singe den Zorn, o Göttin, des Peleiaden Achilleus

Schlimmer noch - leider - 187 Jahre später die Wiedergabe in der vielbenutzten Reclam-Ausgabe von Roland Hampe (1979):

Göttin, singe mir nun des Peleussohnes Achilleus unheilbringenden Zorn!

Man sieht, wie die Wucht des Homerischen 5-Wörter-Verses durch die Änderung allein schon der Wortstellung zerstört wird. Das Emblem, das über dem Ganzen steht, das Giebel-Dreieck des Tempelbaues sozusagen, ist dadurch zertrümmert. Was vorliegt, ist ja nicht die normale Wortstellung im Griechischen. In normaler Alltagsrede stünde der Im-

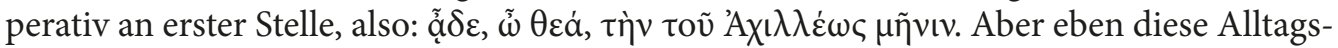
rede vermeidet der Dichter. Er sagt eben nicht: 'Singe den Zorn', sondern er sagt: '[Den] Zorn singe'. Das ist ja kein Zufall. Es ist ihm nicht unbewusst herausgerutscht. Und darum ist es auch nicht etwa unbeachtlich. Wer es als Übersetzer nicht beachtet, erweckt im Leser nicht die angezielte Erwartungshaltung. Das erste Wort der europäischen Literatur - und damit ihr erstes Thema - lautet eben nicht: 'Singe' oder 'Göttin', sondern es lautet: $\mu \tilde{\eta} v ı$.

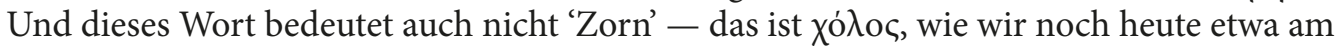
Begriff 'Choleriker' sehen, - sondern es bedeutet 'Groll', wie wir gleich sehen werden. Die treffende Übersetzung ist daher:

„Den Groll singe, Göttin, des Peleïaden Achilleus!“

Das ist nicht etwa erst meine Erkenntnis. Schon Schadewaldt war 1975 in die richtige Richtung gegangen mit der Wiedergabe:

„Den Zorn singe, Göttin, des Peleus-Sohns Achilleus.“

Noch besser die Wiedergabe des amerikanischen Princeton-Professors Robert Fagles von 1990:

"Rage - Goddess, sing the rage of Peleus' son Achilles.“

Falsch ist allerdings immer noch die Wiedergabe des Anfangswortes. Es ist eben nicht 'Zorn' oder 'rage', also 'Wut, Zorn, Rage'. Durch diese Fehlübersetzung wird die angezielte Erwartungshaltung des Hörers oder Lesers geradewegs in die falsche Richtung gelenkt: Er sieht von Anfang an einen zornerfüllten, wütenden, in steter Rage befindlichen Achilleus vor sich. Aber Achilleus ist nicht fast 19 Ilias-Gesänge (also in der Zeitstruktur der Ilias fast 27 Tage lang: Latacz, BK, Prolegomena, S. 152, Abb. 1) - in Rage! In Rage gerät er im ersten Gesang, in der Auseinandersetzung mit Agamemnon, aber dort ist dann dem-

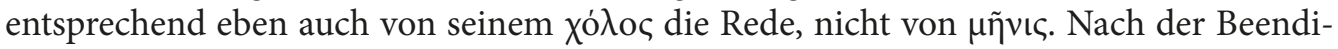
gung dieses wütenden Disputes noch im gleichen ersten Gesang jedoch ist Achill nicht die folgenden fast 19 Gesänge hindurch der wütende Zähneblecker, sondern ein Mensch, der sich zurückgezogen hat in tiefem Groll (grudge). Immer wieder taucht in diesen fast 19 Gesängen als Achills Zustandsbeschreibung das Verb $\mu$ үvi $\omega$ auf, das das Anfangswort der Ilias, $\mu \tilde{\eta} v \iota \varsigma$, das dort erst Programm war, nun in erlebbare Handlung umsetzt. - Und 
wenn Achilleus dann schließlich doch, im 19. Gesang, Vers 75, seinen selbstgewählten Rückzug vom Kampfe aufgibt, da wird das ausgedrückt wiederum durch $\mu \tilde{\eta} v ı \varsigma: ~ \mu \tilde{\eta} v ı v$

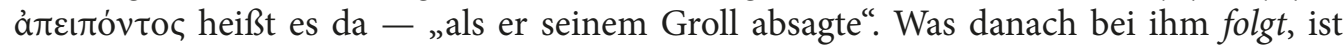
wieder etwas anderes: nicht mehr der nagende Groll wegen der Demütigung - nein, es ist der flammende Trauer- und Rachezorn wegen des Verlusts des Freundes Patroklos ein Affekt, den wir zwar auch als 'Zorn' bezeichnen mögen, der aber mit dem spontanen Jähzorn im Disput mit Agamemnon im ersten Gesang auch wieder nichts zu tun hat. Wir haben nur kein rechtes deutsches Wort dafür: Es ist ein verbissener, in sich erstarrter, aber immer wieder neu aufschäumender traurig-verzweifelter Ingrimm! (darauf kommen wir zurück). - Das alles heißt: Wir tun nicht recht daran, wenn wir diese drei Varianten eines Unmutgefühls (Zorn, Groll, Ingrimm) ohne Beachtung der sprachlichen Differenziertheit des Originals in barbarischer Vereinfachung alle drei schlicht mit dem gleichen Ausdruck 'Zorn' benennen.

Nun noch einmal zurück zum Prooimion! Wir sehen: Am Ende des ersten Verses des Prooimions, in betonter Stellung also, steht Achilleus, und am Ende des letzten Verses des Prooimions, wiederum in betonter Stellung, steht erneut Achilleus. Nach der Antwort, die Achill auf unsere erste Frage geben wird, auf die Frage nach seiner Rolle in der Ilias also, auf diese Antwort brauchen wir danach nicht lange mehr zu warten. „Die Hauptrolle!“ wird er sagen. Vielleicht werden wir einwenden: „Nun ja, Achill - so kündigt Dich das Programm des Dichters an: Aber spielst Du sie dann im Verlauf des Epos wirklich, diese angekündigte Hauptrolle?" - Wenn er hier bei uns stünde und zeichnen könnte, würde er uns die Antwort darauf wohl in Form dieser Graphik geben (Abb. 1).

Hier sehen wir die 24 Gesänge der Ilias vor uns. Ihre Struktur wird bestimmt durch zwei Handlungsebenen: die der Menschen (obere horizontale Linie) und die der Götter (untere horizontale Linie). Beide Linien ziehen sich getrennt durch die ersten 18 Gesänge der Ilias hindurch. Die obere zeichnet Achilleus' Groll nach, die untere das Versprechen des Zeus - jenes Versprechen des Zeus im ersten Gesang, diesem Groll Genugtuung zu verschaffen $(1,523-527)$. Im 19. Gesang dann vereinigen sich die beiden Linien, vereinigen sich zu nur noch einer: Denn im 19. Gesang, Vers 75, beendet Achilleus seinen Groll, und damit zugleich ist das Zeus-Versprechen eingelöst. Jetzt gibt es nur noch éine Handlungslinie. Auf ihr arbeiten Menschen und Götter gleichermaßen auf ein gemeinsames Ziel hin: auf die Bereinigung der Folgen, die aus Achilleus' Groll inzwischen erwachsen sind, vor allem der wichtigsten Folge: Tod des engsten Freundes des Achilleus: Patroklos. Diese Bereinigung besteht in Achills Rache, und diese Rache wiederum ist Hektors Tod. In den Gesängen 19 bis 21 steuert die Handlung unaufhaltsam auf diesen Tod Hektors zu. Im 22. Gesang ist dann das Ziel erreicht: Hektor fällt - unter den Händen des Achilleus. Mit Hektors Fall ist aber auch Troias Fall vorweggenommen. Wir wissen: Jetzt wird Troia nichts mehr retten können. Der 23. und der 24. Gesang sind demgemäß das Ausrollen der Woge: Im 23. Gesang richtet Achilleus in wiedererlangter Ruhe und Gelassenheit, wenn auch voller Trauer, die von der Tradition verlangten Leichenspiele für seinen Freund Patroklos aus, und im 24. Gesang gibt er - mit seinem eigenen Schicksal vor Augen versöhnlich geworden - Hektors Vater Priamos den Leichnam seines Sohnes Hektor - die Trophäe also, die er eigentlich behalten wollte - er gibt sie dem Vater des Todfeinds zurück!

Aus dieser Übersicht wird evident, dass in allen 24 Gesängen der Ilias tatsächlich Achill es ist, der die Hauptrolle spielt. Es dreht sich alles um ihn. Auch dort, wo es vor- 


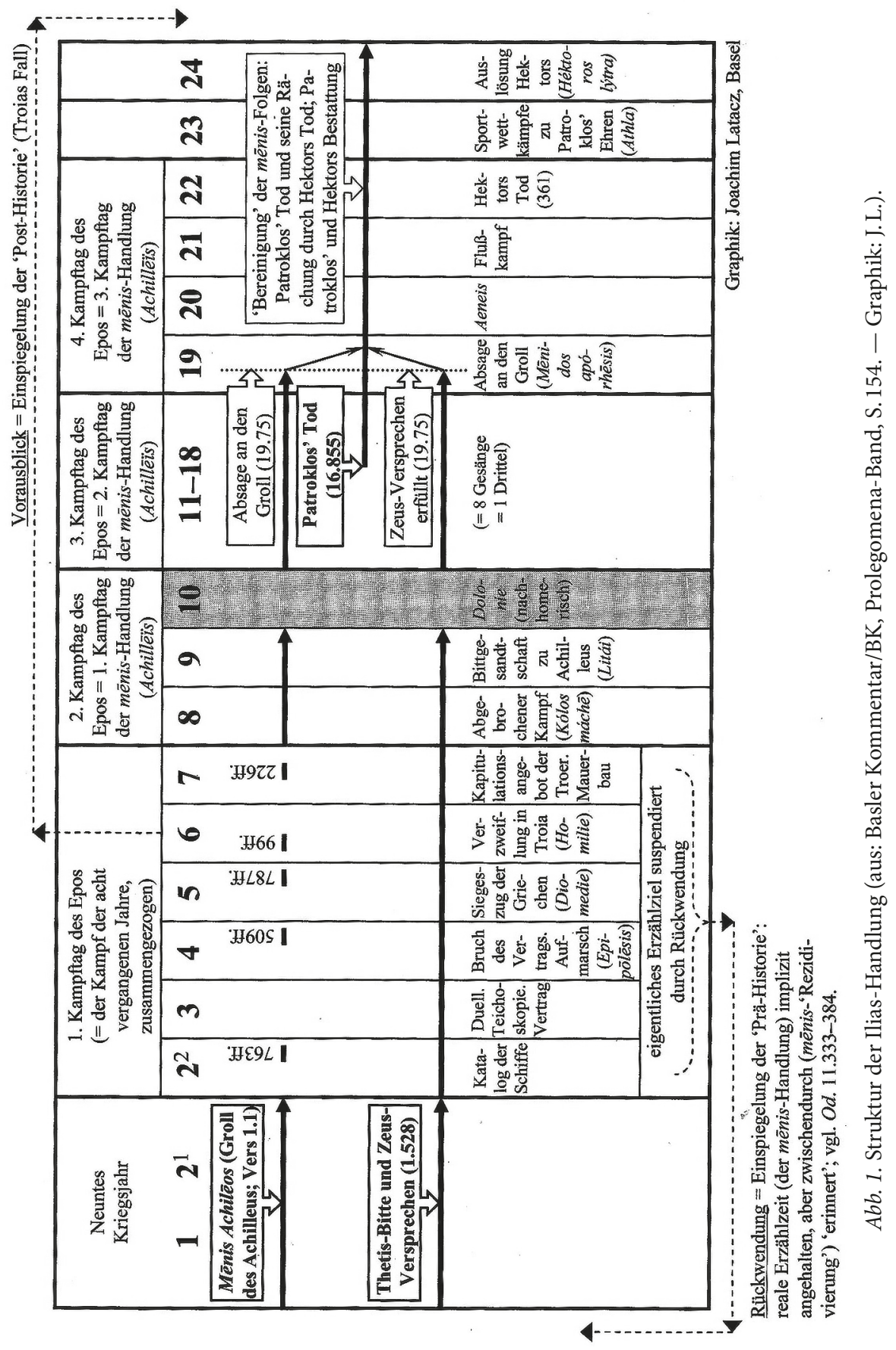


dergründig nicht so aussieht, in den Gesängen 3 bis 18. Das sind die Gesänge, in denen Achilleus am Kampfe gar nicht teilnimmt, in denen er also - abgesehen vom 9. Gesang, auf den wir später zurückkommen - gar nicht auf der Bühne steht. Aber gerade dadurch ist er auch da die Hauptperson. Denn immer wieder in diesen Gesängen, in denen er zurückgezogen in seiner Hütte grollt, wird von anderen Figuren der Handlung mit Bedauern und betrübtem Nachdruck darauf hingewiesen, dass alles, was jetzt geschieht: diese ganze furchtbare Anstrengung der Griechen, von den Troianern nicht bis ins Meer zurückgedrängt zu werden - immer wieder also wird in dieser Handlungsphase darauf hingewiesen, dass all das nicht geschehen würde, wenn Achilleus da wäre, wenn er also nicht grollte. Der Groll des Achilleus ist also gerade da, wo er gar nicht wirksam zu sein scheint, weil er nicht sichtbar wird — gerade da ist er am wirksamsten. Achills Groll, seine Passivität also, wird hier nicht vergessen, sondern ständig bewusst gehalten. Dadurch entsteht höchste Spannung: „Wird Achilleus wieder teilnehmen? Wenn ja, wann endlich wird er denn nun wieder teilnehmen? Denn ohne ihn geht es doch nicht! Das sehen wir doch gerade! Wann also?" Indem der Dichter den Hörer oder Leser derart auf die Folter spannt, lässt er untergründig unsere Aufmerksamkeit immer auf Achill gerichtet sein. So macht er die Aktionslosigkeit Achills zur stärksten Aktion Achills.

Wir sehen: Achill ist vom ersten bis zum letzten Gesang, vom ersten bis fast zum letzten Vers der Ilias $(24,779)$ tatsächlich das eigentliche Thema der Ilias. Nicht die Geschichte von Ilios, die in der Sagenchronologie rund 40 Jahre umfasst, auch nicht der Krieg um Ilios mit seinen 10 Jahren Dauer - das alles ist nur Schauplatz, Hintergrund, Kulisse. Natürlich wird diese Kulisse im Werkverlauf implizit allmählich detaillierter, profilierter, dichter. Im Kern jedoch ist das, worum es geht, nicht sie, sondern — Achilleus.

\section{II}

Unsere erste Frage an Achilleus ist damit beantwortet. Wir wenden uns der zweiten zu. „Was ist die Ursache für Deinen Groll?" Die Antwort wäre wohl: „Das wird sich zeigen, wenn ihr zuvor fragt, wer ich bin!“ - Gehen wir diesem Wink ein Stückweit nach! In dem leidenschaftlichen Disput des Werkbeginns zwischen ihm und Agamemnon beendet Achilleus den ersten Teil des Wortgefechtes mit den Worten (1, 243-244):

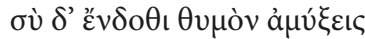

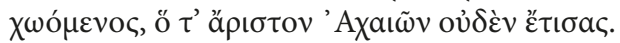

„Du wirst dir im Innern dann den Sinn zernagen

vor Wut, dass du den Besten der Achaier nicht geehrt hast! “

(Übers: J.L.)

Achilleus bezeichnet sich also selbst als den 'Besten der Achaier'. Er wird das noch zwei weitere Male in der Ilias tun. Ist das Größenwahn? Weit gefehlt! Er sagt nur das, was alle von ihm sagen, nicht nur die Achaier, sondern auch die Troer und - mehr noch auch die Götter! Dass es sein engster Freund Patroklos von ihm sagt $(16,271)$, das ist verständlich. Dass es aber z.B. auch Glaukos von ihm sagt, einer der beiden Anführer der troischen Bundesgenossen aus Lykien, und zwar zu Hektor sagt, dem Oberkommandierenden der troischen Verteidigungsarmee, und zwar als eine Selbstverständlichkeit sagt, die unwidersprochen bleibt, so dass er damit also offensichtlich eine communis opinio wiedergibt $(17,164 f$.) - das ist signifikant! Und wenn wir daraufhin die ganze 
Ilias durchsehen, erkennen wir sogleich, dass ein Zweifel, wer der Beste der Achaier ist, bei niemandem besteht. Ein paar Beispiele: Zuerst der Dichter selbst, also das auktoriale Lenkungs-Urteil: Achilleus sei ein „göttlicher“ Mann, sagt Homer (16, 798), ein Mann, der „dem Zeus lieb“ ist $(24,472)$, usw. usf. — Dann die eigenen Leute, die Achaier. Odys-

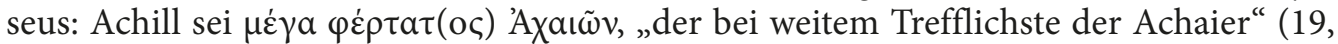
216). Nestor: Achilleus sei ein $\dot{\varepsilon} \sigma \theta \lambda$ ó $\varsigma$, „ein tüchtiger Vornehmer“ $(11,665)$. Und sogar Agamemnon gibt zu: Achill sei ảyäóc, „ein tüchtiger Edelmann“ $(1,131)$. — Dann die Gegner, die Troianer. Hektor zu Achill: „Ich weiß, dass du ein tüchtiger Vornehmer bist, und ich viel geringer bin als du!“ $(20,434)$; Aineias: „Unmöglich ist es, gegen Achilleus, wenn man ein Mensch ist, zu kämpfen“ (20, 97-98). Und schließlich Priamos selbst, Troias König: „Voller Staunen betrachtete er den Achilleus, wie groß er war und wie schön: Den Göttern glich er, verglich man's!“ (24, 629-630). - Und als Höhepunkt die Götter: Zeus: „Achilleus ist ein Fürst, vor dem noch ganz andere [als Hektor] zittern“ (17, 205). Hera: „Stehen wir doch Achilleus helfend zu Seite, auf dass er weiß, dass die Besten der Götter ihn lieben" (20, 120-122), und auch Poseidon, der eigentlich AchilleusFeindliche, zum Troer Aineias: „Achill ist zugleich stärker als du und den Unsterblichen lieber!“ (20, 334). Wir könnten diese Worte der Anerkennung, ja, der uneingeschränkten Bewunderung und nahezu Vergöttlichung noch lange fortsetzen: Die Stellen ohne explizite Namensnennung sind ja noch viel zahlreicher. Es ist kein Ruhmesblatt der HomerForschung, dass sie eine vollständige, klare und übersichtliche Zusammenstellung der werk-internen Urteile über Achilleus bis heute nicht zustande gebracht hat. Aber schon aus den wenigen Zitaten ist klar, dass Achilleus derjenige Held der Ilias ist, dem die volle Sympathie des Dichters gilt; denn der Dichter ist es ja, der den Achilleus nicht nur in eigener Person, also auktorial, rühmt, sondern der alle diese Rühmungen seinen Handlungsfiguren in den Mund legt.

Worauf basiert aber dieses exzeptionell positive Urteil über Achill? Er sagt es oftmals selbst, und niemand bestreitet es: Zunächst und zuallererst beruht es auf seiner überragenden Qualität als Kämpfer. Gleich im ersten Gesang legt er die Karten auf den Tisch. Zu Agamemnon (1,152-168):

„Doch nicht der Troer mit den langen Lanzen wegen habe ich den Weg genommen hierher zum Kampfe, denn mir sind sie ja nun gar nicht schuld an irgend etwas:

$[\ldots]$

Nein — dir, zutiefst schamloser Mensch, sind wir gefolgt, damit du Freude hättest

die Ehre wiederzugewinnen suchend Menelaos und dir selbst, Hundsauge,

von den Troianern! Darum scherst du dich jedoch kein bisschen, sorgst dich nicht drum!

$[\ldots]$

Nicht hab' ich jemals ein Geschenk von gleichem Wert wie deins, wenn die Achaier

im Troerland vernichtet haben eine wohlbewohnte Stadtburg;

o nein, den größren Teil des heftig tobenden Gewühls im Kampfe,

den dürfen meine Arme leisten; doch sobald die Beuteteilung ansteht,

fällt dir die weitaus größre Ehrengabe zu - und ich darf mit der kleinen, feinen

zurück zu meinen Schiffen gehn, nachdem ich müde bin vom Kämpfen!“

(Übers.: J.L.)

Das eigentliche Kraftzentrum im Kampf um Troia ist also Achilleus. Agamemnon, der Oberfeldherr, sitzt in seinem Feldherrnzelt, lässt Achilleus kämpfen und nimmt die besten Stücke aus der Beute, die Achill ihm mitbringt, hoheitsvoll entgegen. 
Das also ist Achilleus! Ein Unersetzlicher! Und diesen Unersetzlichen - der überdies an Adelsrang dem Agamemnon mindestens gleichwertig ist, denn beide sind Urenkel bzw. Ur-Urenkel des Zeus, und Achills Mutter ist noch dazu eine Göttin, die Meeresgöttin Thetis - diesen Unersetzlichen also hat Agamemnon in der Auseinandersetzung des Werkbeginns, im 1. Gesang, vor dem gesamten Heer der achaiïschen Allianz, coram publico, abgekanzelt wie einen - so wird Achill es später sagen - wie einen ảov́ $\varphi \eta \lambda$ oc,

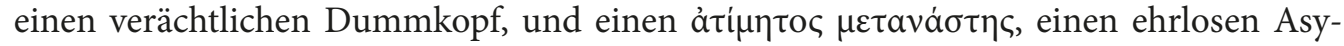
lanten $(9,648-649)$. Er hat ihm nicht nur sein wohlverdientes Ehrgeschenk, sein $\gamma \dot{\varepsilon} \rho a \varsigma$, die Kriegsgefangene Brisēis, vor versammelter Mannschaft persönlich wegzunehmen gedroht - ein Geschenk, das gar nicht er ihm gegeben hatte, sondern das ganze AchaierHeer, - sondern er hat ihn auch verächtlich weggejagt (1, 173-176):

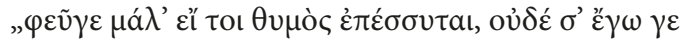

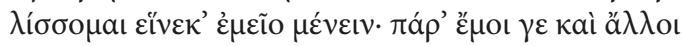

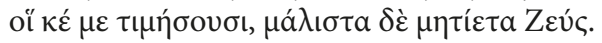

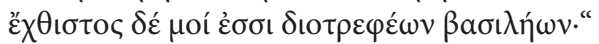

„Verzieh dich nur, wenn dir der Sinn so danach giert! Ich flehe dich bestimmt nicht

kniefällig an, zu bleiben, mir zulieb! Bei mir sind schließlich auch noch andre,

die mir die Ehre rückerobern werden - und vor allen andern Zeus, der weise. -

Bist der verhassteste mir ja im Kreis der Könige, der zeusgenährten."

(Übers.: J.L.)

$\varphi \varepsilon \tilde{v} \mathcal{E}^{\prime} \mu \alpha^{\prime} \lambda^{\prime}$ - wer nur ein wenig Neugriechisch kann, der versteht die Tiefe der Beleidigung sofort, die darin liegt: $\varphi \dot{\gamma} \gamma \varepsilon$, $\varphi v \dot{\gamma \varepsilon ! ~-~ s o ~ s c h e u c h t ~ m a n ~ h e u t e ~ n o c h ~ e i n e n ~ h e r-~}$ renlosen Hund fort, der am Tisch um Essensreste bettelt. Ich denke, wir können auch heute noch erfühlen, wie unermesslich die Demütigung ist, die dem Achilleus da von Agamemnon vor dem gesamten Heere widerfahren ist! Nicht nur jedem General, der vor seinen Soldaten, sondern auch schon jedem Schüler, der so vor der ganzen Klasse heruntergemacht würde, würde die Scham- und Zornesröte ins Gesicht steigen — und die Wunde, die da geschlagen wurde, säße tief, sehr tief, für lange Zeit! Sie würde schwären und schwären! Und was daraus entstünde, wäre eben das, was sich in die Seele des Achilleus, je länger er darüber nachdenkt, immer tiefer einfrisst: die $\mu \tilde{\eta} v ı \varsigma$, der Groll!

Ist es also wirklich der Verlust des Mädchens Briseis, der Achilleus zum Boykott veranlasst? Gewiss - auch das spielt mit; an einer Stelle sagt er selbst: „Ich hatte sie von Herzen lieb" $(9,342-343)$. Doch das Entscheidende ist nicht das Mädchen, sondern die unerhörte Demütigung, die mit ihrer Wegnahme verbunden ist. Denn was da geschehen ist, ist das Schlimmste, was einem Adligen von Rang und Leistung des Achilleus überhaupt geschehen kann: Weggenommen wurde ihm nicht nur das Mädchen - weggenommen

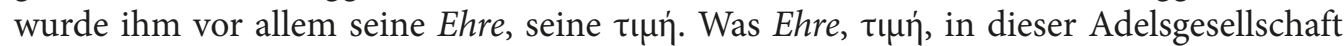
eigentlich bedeutet, ist für uns heute nicht mehr sofort nachfühlbar. Ihr Verlust ist die soziale Vernichtung!

Das also ist der eigentliche Grund für Achills Rückzug vom Kampf und für seinen Boykott. Die vielen Homer-Experten, die dem Achilleus vorwerfen zu dürfen glauben, dass er danach einsam zum Meeresstrande geht und weint und seine Mutter, die Meeresgöttin Thetis, aus den Fluten zu sich ruft - und die sich lustig machen, die Experten, über diesen Göttersohn und Superhelden: da sitze er nun da - und flenne wie ein Kind, dem man sein Spielzeug weggenommen hat — sie alle haben die wirkliche Bedeutung 
dessen, was dem Achill da zugemutet wurde, nicht verstanden. Die Folgen dieses Nichtverstehens aber sind verhängnisvoll: Die Ilias als Ganzes wird nun nicht mehr recht verstanden.

\section{III}

Um diesen Fehler nicht zu machen, stellen wir Achilleus jetzt die dritte Frage: „Warum lehnst Du das Wiedergutmachungsangebot ab?" Ich glaube, die Antwort darauf würde nunmehr kürzer sein. „Ihr seht doch nun, wie ich behandelt wurde - und wie ich seitdem darunter leide! Versteht ihr nicht, wie mir zumute ist, als Agamemnon mich durch Mittelsmänner wieder zurückzugewinnen sucht? Versteht ihr nicht, wie mir zumute ist, wenn er mir sagen lässt, auf welche Weise er die Wunde, die er mir geschlagen hat, heilen will?" Etwa in dieser Art würde Achilleus wohl zu uns sprechen. Betrachten wir daraufhin Agamemnons Wiedergutmachungsangebot - das Agamemnon natürlich notgedrungen macht, weil er Achilleus, den Weggejagten, jetzt verzweifelt dringend wieder braucht betrachten wir also dieses Angebot genauer. Was umfasst es?

Odysseus zählt die Offerte auf. Teil 1. Jetzt sofort: 7 nagelneue Dreifüße, 10 Talente Gold, 20 Silberbecken, 12 Rennpferde bester Zucht, 7 Frauen aus Lesbos (diese galten als die schönsten - Topmodels sozusagen), dazu die - wie er betont: unberührte - Briseis. Und später, Teil 2, nach der Eroberung Troias (die natürlich Achill selbst leisten soll) eine Schiffsladung Gold und Bronze, die 20 schönsten Troerinnen nach Helena, und schließlich - eine seiner eigenen drei Töchter zur Ehefrau, mit der Mitgift von 7 Städten!

Was für ein Angebot! Die Homer-Interpreten, allen voran erstaunlicherweise die deutschsprachigen, waren sich lange Zeit und sind sich weitgehend noch heute einig: Dieses Angebot kann man nicht verschmähen. Annehmen hätte hier Achilleus müssen, hieß und heißt es, die Ablehnung eines derart generösen Angebots sei übertriebene Härte, kindischer Trotz, ja sogar "das tragische Urmotiv der überschrittenen Grenze“ (so Lesky 1967, 95). Was sich in solchen rügenden Zumutungen enthüllt, ist ein tiefes Unverständnis. Die Interpreten, die so reden, stellen sich auf eine Stufe mit dem, der diese Angebote macht: mit Agamemnon - mit einem Agamemnon, der nicht das Mindeste begriffen hat, der nicht verstanden hat, dass es hier nicht um Materielles geht, sondern um eine Verletzung, die, wenn überhaupt, nur der Verletzer selbst, persönlich, nicht durch Mittelsleute, Einsicht zeigend, wieder heilen könnte! Das materielle Angebot des Feldherrn - Millionen sozusagen, und dazu ein riesenhafter Harem von insgesamt 29 Frauen (weil dieser Mann, dieser Agamemnon, so wie er selbst denkt, immer noch im Wahne lebt, Achilleus gehe es um Frauen!) - dieses Angebot kann Homers Achilleus gar nicht anders denn als rüden Kaufversuch verstehen - Geld gegen Ehre! - und damit als eine weitere Entehrung! Es erscheint fast paradox, dass nicht nur britische, sondern vor allem amerikanische Homer-Interpreten Achill in diesem Punkte nicht nur besser, sondern sogar voll verstehen: Der britische Homeriker Oliver Taplin etwa formulierte 1986: „Achilles, far from being satisfied, takes this as adding insult to injury" (Taplin 1986, 16). Und der amerikanische Homer-Interpret Mark W. Edwards hat die Dinge energisch zurechtgerückt, als er 1987 formulierte: „It is Agamemnon's humiliation that Achilles desires, not an increase in his stature. Gifts of that value would reaffirm in the strongest way Achilles' subordinate status to Agamemnon." (Edwards 1987, 234ff.). Und ich selbst habe 1995 formuliert: „Die Aufzählung wird in einer Art 
Häufungsrausch bis zu einem Gewicht gesteigert, das Achill erdrücken soll“ (jetzt Latacz 2014, 328 Anm. 133).

Dass der Dichter es genau so gemeint hat, macht er selbst klar, wenn er den Agamemnon diese Warenliste, die sein Abgesandter Odysseus hier vor Achill nur wiederholt, bei ihrer ersten Aufzählung abschließen lässt mit einem triumphierenden: „Das alles biete ich ihm an — aber: $\delta \mu \eta \theta \dot{\eta} \tau \omega$ ! — Beugen soll er sich!“ $(9,158)$. Den Odysseus als Abgesandten lässt der Dichter dann diese ganze Aufzählungsrede Agamemnons vor Achilleus wiederholen - dieses letzte „Beugen soll er sich“ aber — das lässt er den Odysseus verschweigen! Deutlicher kann Homer nicht klarmachen, dass er diesen ganzen Gabenkatalog nicht nur als Bestechungsversuch verstanden wissen will, sondern auch als Aufforderung zu definitiver Unterwerfung.

Warum Achilleus ablehnen muss, ist danach klar: nicht aus Trotz, sondern aus Selbstachtung! Darum bleibt er allem Zureden der Abgesandten gegenüber unzugänglich — so wie es der Triptolemos-Maler um 480 auf dem berühmten Krater aus Athen mit sicherem Griff ins Bild gesetzt hat (Abb. 2)

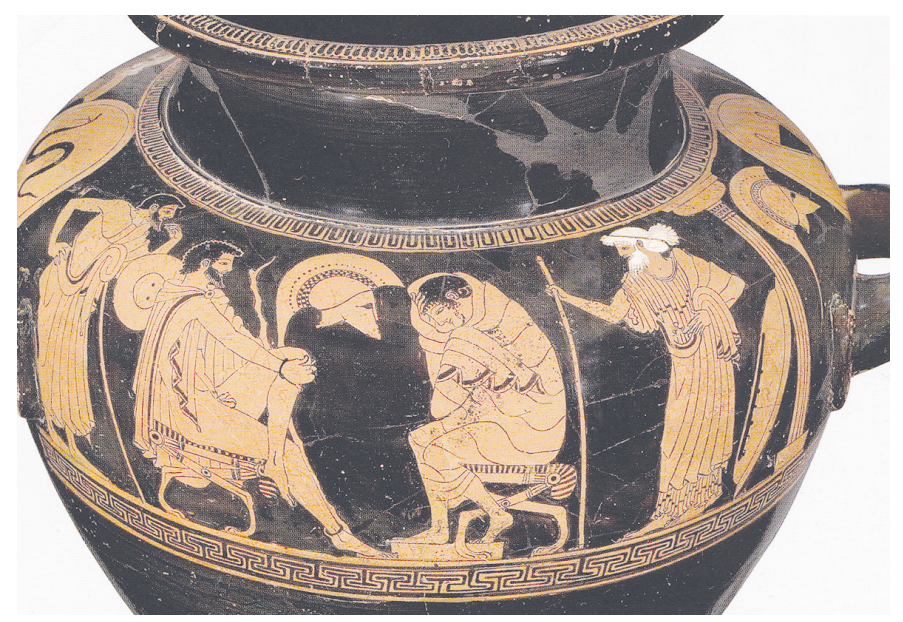

Abb. 2. Odysseus und Achilleus. Rotfig. Weingefäß: Athen um 480 v. Chr. Werk des Triptolemos-Malers (Antikenmuseum Basel und Sammlung Ludwig. Inv. BS 477).

Odysseus, links, sitzt entspannt und redet - sich seines Erfolgs ganz souverän gewiss - Achilleus sitzt, fest in seinen Mantel eingehüllt, ihm gegenüber, schweigt und drückt mit seiner ganzen gedrückten, ja gequälten Haltung aus: „Ich kann nicht!“ Im Text heißt es: „Agamemnon wird mich niemals überreden können, bevor er mir nicht die in meinem Innern schmerzende Kränkung ganz gebüßt hat!“ (9, 386f.). Dieses 'ganz' sagt alles. Agamemnon muss am Boden liegen, konkret: er muss - den Untergang vor Augen - persönlich, vor dem ganzen Heer - wie damals bei der Kränkung - dem Achilleus Abbitte leisten! Solange er das nicht tut, hat er seinen damaligen Fehler, Achills Demütigung von Anfang an, noch immer nicht erkannt und diesen Fehler also auch nicht offen vor Achilleus eingestanden. Darum kann Achilleus die Gesandten am Schluss auch nur bescheiden: „Sagt den Edlen der Achaier, sie mögen einen andren Rettungsplan ersinnen - ich grolle weiter!“ $(9,421-423)$. 
Hier werden manche fragen: Schön — aber warum nimmt er dann das gleiche Angebot zwei Tage später, im 19. Gesang, widerspruchslos an? Leider können wir diese fast groteske Szene im 19. Gesang hier nicht ausführlich interpretieren. Nur soviel: Hier hat sich die Situation total gewandelt! Denn zwischen diesen beiden Angebotsszenen ist Patroklos gefallen. Patroklos ist Achills engster älterer Freund seit Jugendtagen, wie wir im 11. Gesang erfahren haben. Sie sind zusammen aufgewachsen, sie haben alles gemeinsam getan (24, 3-9). Patroklos ist nahezu Achills zweites Ich. Zwischen ihnen besteht eine tiefe innere Bindung. Sie ist so stark, dass Achill nach Patroklos' Tod vor Trauer und Verzweiflung alles andere für nicht mehr wichtig hält. Als Agamemnon ihm im 19. Gesang das gleiche Angebot wie 2 Tage vorher nun selbst in der Versammlung macht - allerdings in höchst gewundener Form und wieder nicht mit einer offenen, aufrichtigen, an ihn selbst gerichteten Entschuldigung, sondern mit einer weitausholenden Geschichte über die Macht von Atē, der Göttin der Verblendung -, da hört sich Achill all das mit halbem Ohr ergeben an und sagt dann nur, nicht ohne Ironie (19, 146-150):

„Atride, majestätischster! Du Herr der Männer Agamemnon!
Die Gaben - nun, ob du sie geben willst, wie es der Brauch ist,
oder behalten, steht bei dir. Jetzt aber wollen wir auf Kampflust sinnen,
ganz schnell! Nicht langes Reden tut uns nämlich not, hier sitzend,
und auch nicht Zeit verschwenden. Noch ist ungetan das große Werk ja!“

(Übers. JL)

Das „große Werk“ aber ist die Rächung von Patroklos’ Tod, die Rächung an Hektor! Angesichts dieses Zieles rückt die Geschenke-Frage und mit ihr der ganze Streit mit Agamemnon für Achill weit in den Hintergrund. Mag Agamemnon ihm die Geschenke geben oder nicht - das ist ihm nunmehr gleich - womit er kundtut, dass er sie nun nicht mehr als eine Bezahlung für seinen Wiedereintritt in den Kampf ansieht, damit aber auch nicht mehr als Kompensationsversuch für die ihm zugefügte Kränkung. Und als Agamemnon auf den Geschenken besteht, da lässt er es halt ungeduldig geschehen, dass sie feierlich hergebracht werden, weil das nun einmal die Voraussetzung für den Aufbruch zum Rachezug des Heeres ist. Eine Versöhnung ist damit für ihn nicht verbunden. Eine wirkliche Versöhnung mit Agamemnon ist für ihn ohnehin nicht denkbar ganz grundsätzlich! Die beiden Charaktere sind unvereinbar, denn sie stehen in zwei verschiedenen Welten.

\section{IV}

Damit kommen wir zur vierten und letzten Frage: „Was ist für Dich der höchste Wert?" In seiner Antwort darauf würde Achilleus wohl als erstes jene Devise zitieren, die ihm sein Vater Peleus beim Abschied mit auf den Weg nach Troia gegeben hat $(11,784)$ :

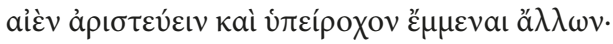

„Stets sich als Bester bewähren und trefflicher sein als die andren!“

(Übers.: JL)

Das lässt Homer im 6. Gesang wortgleich auch den Lykier Hippolochos zu seinem Sohn Glaukos bei dessen Aufbruch nach Troia sagen $(6,208)$. Daher wissen wir, dass es 
ein altes, traditionelles Adelsmotto war - Bestandteil der Erziehung eines jungen Adligen. Was aber bedeutet dieses „Stets Bester sein“? Welche Werte werden da verlangt?

$\mathrm{Da}$ ist zunächst der Wert der Vorzüglichkeit im Kampfe. Das ist kein rein physischer Wert. Nicht körperliche Kraft allein ist damit gemeint. Die ist Voraussetzung. Aber die hat auch ein guter Boxer - etwa wie der Boxer Iros in der Odyssee: stark, aber geistig eher kärglich. Vorzüglichkeit im Kampf bedeutet für Achilleus, wie wir aus zahlreichen IliasStellen belegen könnten, über das Körperliche hinaus vor allem Mut, Entschlossenheit, Zähigkeit, aber auch die Gabe, andere mitzureißen und sie bei der Stange zu halten, also kurz: überlegene, aber auch überlegte Führungsstärke. Das nächstliegende Gegenbeispiel wäre Aias: ungeheuer stark und zäh, aber nicht annähernd so selbstreflektiert wie Achilleus. Darum heißt es ja in der Ilias und auch sonst in der griechischen Literatur stets von Aias: Er war der beste Kämpfer - nach Achilleus.

Der kurze Vergleich Achilleus : Aias hat uns aber bereits auf den entscheidenden Unterschied eingestellt: Achilleus haut im Normalzustand nicht wild drauflos, nur um draufloszuhauen, sondern er fragt zuerst nach dem Warum, d. h. er überlegt zuerst, um welches Ziel es geht. Wir haben gehört, dass er sich für den Zug nach Troia hat anwerben lassen, nicht, um ein großer Kriegsheld zu werden und möglichst viele Menschen totzuschlagen, sondern weil er den Atriden helfen wollte, ihre durch Helenas Raub verlorene Ehre wiederzugewinnen $(1,152-160)$. Damit kommen wir noch einmal auf den Wert der

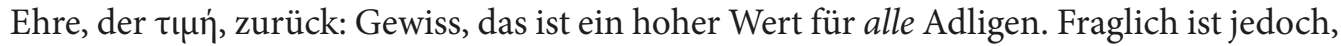
ob irgendeine von Homers Figuren diesen Wert so tief verinnerlicht hat wie Achilleus. Man sieht das sehr leicht daran, dass die Homer-Interpretation gerade ihm immer wieder ein angeblich übertriebenes Ehrbewusstsein vorgeworfen hat. Darin zeigt sich, wie ich glaube, wiederum nur, dass die Interpreten Homers Achilleus nicht gewachsen sind. Für sie hat Ehre nicht den gleichen 'Stellenwert' wie für Achill. Sie wären also anscheinend bei Verletzung ihrer eigenen Ehre eher zu Kompromissen bereit.

Wir können hier leider nicht den Unterschied zwischen adligem und bürgerlichem Ehrbegriff herausarbeiten, schon gar nicht mit Bezug auf den adligen Ehrbegriff der Zeit Homers! Dazu müssten wir den ganzen fundamentalen historischen Gesellschaftsunterschied analysieren. Aber wir können feststellen, dass Achilleus den Mann, der sich die Ehre nehmen lässt - so wie Agamemnon es bei ihm versucht hat - dass er diesen Mann

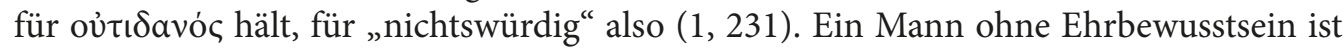
für ihn eine Null. Es ist merkwürdig, dass viele Interpreten das nicht wenigstens im Ansatz verstehen. Im Wehrstrafgesetz der Bundesrepublik Deutschland steht in $\$ 31$ (1) „Wer einen Untergebenen entwürdigend behandelt [...], wird mit Freiheitsstrafe bis zu fünf Jahren bestraft." Demnach würde ein Agamemnon, so wie er den Achill behandelt hat, heute im Gefängnis landen. Und wie würden sich die Interpreten, die sich über Achills angeblich „sturen“ Ehrbegriff ereifern - wie würden sie sich fühlen, wenn sie z. B., wie die Gesetzesformel heißt, „unehrenhaft entlassen“ würden, oder gar, wenn ihnen in einem Rechtsstreit die „bürgerlichen Ehrenrechte“ aberkannt oder wenn „ehrenrührige Behauptungen“ über sie aufgestellt würden? So fremd ist also der Wert „Ehre“ auch uns noch nicht. Achilleus aber verkörpert den Wert „Ehre“ rein, nahezu als platonische Idee. Denn er versteht, was Harald Weinrich einmal so auf den Punkt gebracht hat: „Denn die Ehre kennt kein Mehr oder Weniger; man hat die Ehre entweder ganz oder man hat sie gar nicht“" (Weinrich 1971, 165). Wer das begriffen hat, so wie Homers Achill, der begreift mit einem Mal die ganze Ilias besser. 


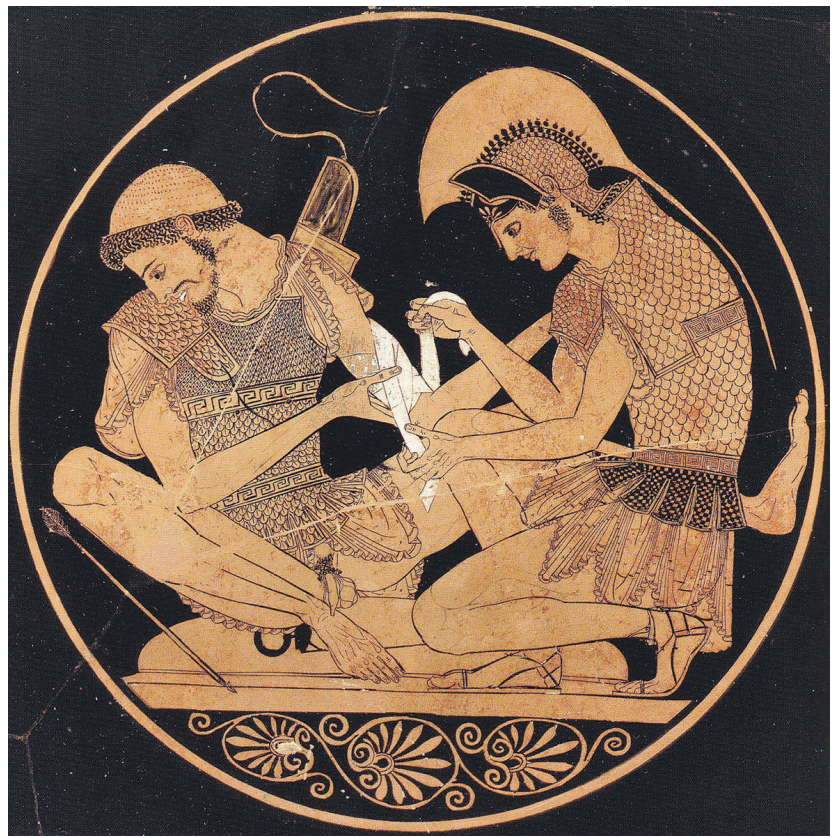

Abb. 3. Achill verbindet seinen verwundeten Freund Patroklos. Schale des Sosias-Malers um 500 v. Chr. Staatliche Museen zu Berlin, Preußischer Kulturbesitz.

Achilleus kennt aber noch viele andere Werte. Die Freundschaft etwa. Was die für Achill bedeutet, hat der Sosias-Maler um 500 offenbar gut verstanden und ins Bild gesetzt.

Viele weitere Werte muss ich übergehen. Ein par Andeutungen wenigstens zum Wert der Wahrhaftigkeit. Bezeichnend — und vielzitiert — sind seine Worte zu Odysseus im 9. Gesang der Ilias:

„Verhasst ist mir der Mann gleichwie das Tor zum Hades,

der eins im Sinn versteckt — und etwas andres ausspricht!“ $(9,312 f$.$) .$

(Übers.: J. L.)

Die Offenheit der Rede, die er selbst dagegen praktiziert, wird ihm von den Interpreten oft als Rücksichtslosigkeit ausgelegt. Er sei hart, brüsk, schroff. Einspruch auch hier! Wer die 85 direkten Reden mit ihren fast 1000 Versen zusammensieht, die Homer dem Achilleus in den Mund gelegt hat (keine Ilias-Figur redet häufiger als er), der kann nicht umhin, statt Schroffheit etwas anderes zu konstatieren: Den Willen zu unbedingter Klarheit. Um eine Sache herumzureden ist für ihn unmöglich, und andere um eine Sache herumreden zu hören ist ihm peinlich. Zwar schlägt er dann verbal nicht zu wie ein Barbar, aber er bleibt in seiner Antwort geradezu eiskalt sachlich - so wie im 9. Gesang gegenüber der großartigen und ästhetisch wunderschönen Rhetorik des Odysseus. Achills Motto ist das des alten Cato: Rem tene, verba sequentur!

Mit dem Stichwort „Härte, Schroffheit" sind wir bei einem weiteren Vorwurf, der gegen ihn erhoben wird (und z. T.schon in der Antike erhoben wurde): In einem Aufsatz eines Kollegen von 1988 hört sich das so an: „entmenschter Held“, „unmenschliche Wildheit“, „der tierisch Verrohte“, „unmenschliche, tierische Verwilderung“ (Effe 1988, 
11-13).- und noch kürzer und auf den Begriff gebracht im allbekannten Label, das Christa Wolf 1983 dem Achilleus aufgedrückt hat, als ein epitheton damnans, wie man gesagt hat (Wülfing 1993, 6): „Achill das Vieh“ (Wolf 1983 passim/Latacz. 1995. 1997, 67 Anm. 1).

Ich gestehe, von einem viehischen Achilleus in der ganzen Ilias nichts zu sehen. Was ich sehe, in jenem Achill, der nach Hektors Tötung seines Freundes Patroklos wie von Sinnen ist vor Schmerz und Trauer, ist ein Achilleus, der verzweifelt versucht, die blutende Wunde, die ihm dieser Tod des einzigen wirklichen Freundes geschlagen hat, irgendwie zu heilen oder mindestens zu lindern, und der aus dieser Verzweiflung heraus das einzige Heilmittel in einer Kompensation durch Tötung so vieler Feinde sieht, wie sein Freund ihm wert war $(21,133 f$.). Man muss jede einzelne Tötungsszene in diesen letzten Gesängen der Ilias genau und immer wieder neu lesen, um das zu verstehen. Hier muss ein einziges Beispiel genügen: die Tötung des Lykaon. Priamos' Sohn Lykaon, von Achill schon einmal verschont, bittet im 21. Gesang erneut um Schonung. Achill lehnt ab. „Früher“, sagt er, „hab” ich viele Troer verschont und nur gefangengenommen, so wie Dich (vgl. 24, 751-754). Jetzt aber, nach Patroklos' Tod kann ich keinen mehr schonen, schon gar nicht einen von Hektors Brüdern." Und dann kommen die Worte $(21,99-113)$ :

„Auf denn, mein Freund, stirb auch du! Warum denn jammerst du so sehr?

Sterben musste auch Patroklos, der doch viel besser als du war!

Siehst du nicht, wie ich selbst bin? so schön und so groß doch!

Sohn eines adligen Vaters - die Mutter sogar eine Göttin - ,

aber auch neben mir steht der Tod und das mächtige Schicksal!

Kommen wird ja der Morgen, der Abend oder der Mittag,

da auch mir einer ausbläst im Kampfe das Leben,

ob nun durch fliegende Lanze oder durch Pfeil von der Sehne!“

(Übers.: J.L.)

Diese Szene hat man als Beleg für unmenschliche Grausamkeit angeführt: Achill der Schlächter. Aber wie spricht Achill denn diese Worte? Spricht er sie triumphierend, jauchzend? Nein - er spricht sie wissend, fast schon tragisch. Er schlachtet nicht ab! Er handelt

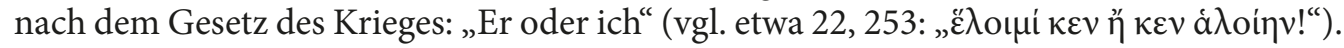
Im Hintergrund steht eine realistische und zugleich traurige Illusionslosigkeit. Das kann von Philologen, die nie im Feld gestanden haben, auch nie verstanden werden. Niemand hat das klarer gesehen als Jonathan Shay, der klinische Psychiater aus Boston, in seinem 1994 erschienenen Buch Achilles in Vietnam: Combat Trauma and the Undoing of Character, das hervorragend besprochen wurde von Sallie Goetsch (University of Michigan/Ann Arbor): Patroklos' Tod - die Stellen im Homer-Text, die das immer wieder neu ausdrücken, machen es überdeutlich klar - war für ihn der Gipfelpunkt, der in seinem Innern alles verändernde Schock, den er erlitten hat in seinem nun fast 10jährigen Kriegserlebnis voll von „terror, horror, grief, and guilt", und dieser Schock, der erfolgt ist nach seiner

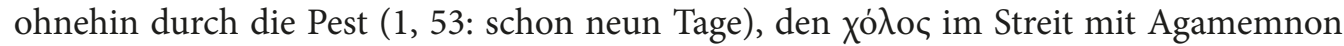
und durch die ihn selbst doch am meisten quälende $\mu \tilde{\eta} v ı$ der letzten 25 Tage, lässt ihn innerlich zusammenbrechen, fast den Verstand verlieren, wie es ihm Apollon im Kreis der Göttter - auch er verständnislos — zornig vorwirft (24, 40ff. ש̃

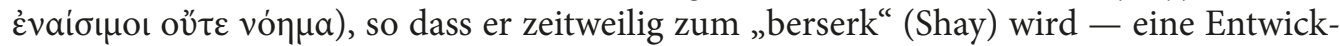
lung, die, wenn der Betroffene nicht von seinem Umfeld verständnisvoll aufgefangen wird (wie hier Achilleus in den letzten Ilias-Gesängen, besonders durch die Maßnahmen des 


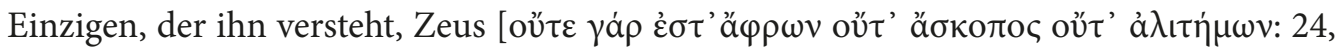
157] zum lebenslangen Trauma führt).

Achill erfüllt das Schicksal - im Wissen, dass ein andrer es an ihm genauso - und schon bald - erfüllen wird. So handelt ein im Kern mitleidender, doch seiner selbst vor tiefem Leid, Selbstanklagen („warum habe ich dich bloß gehen lassen, Patroklos!“), Ingrimm und rasendem Rächungswillen (Leitbegriff: $\mu \varepsilon \dot{v}{ }^{\prime} \varsigma$ mit $\left.\dot{\varepsilon} \mu \mu \varepsilon \mu \omega \dot{\omega} \varsigma, \mu \varepsilon v \varepsilon a i v \omega\right)$ nicht mehr bewusster Vollstrecker.

Viel wäre noch zu sagen - über Achills Freundlichkeit etwa, über seine Ehrfurcht vor den Alten und vor den Göttern - und vieles mehr. Das alles gehört für ihn zum Re-

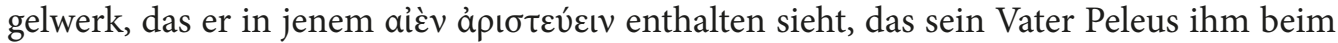
Abschied in Phthia mitgegeben hat $(11,784)$. Achill beherrscht das ganze Regelwerk -

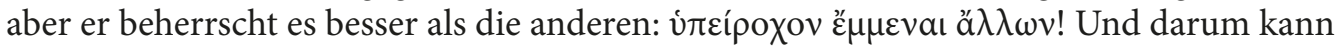
er nicht begreifen und nicht verzeihen, wenn andere gegen dieses Regelwerk verstoßen.

Was aber ist für ihn der höchste Wert? In der Regel lautet die Antwort hierauf: „Ruhm“, $\kappa \lambda \varepsilon \dot{o}$. Ist das richtig? Sieht er selbst das auch so? Letztlich schon. Aber mir scheint, er würde auch hier differenzieren! Darauf führt jene hundertfach diskutierte Aussage, die der Dichter dem Achilleus in seiner Ablehnungsrede im 9. Gesang in den Mund legt. Alle Schätze der Welt, sagt er dort, sind ihm nicht so viel wert wie das Leben. Denn alles Materielle ist ersetzbar, sagt er, aber das Leben eines Mannes kann man nicht erbeuten, dass es wiederkehre, noch packen, wenn es einmal überschritten hat den Zaun der Zähne (9, 408f.).

Und dann kommt die berühmte Alternative: Was ist mehr wert: ein kurzes, aber ruhmreiches Leben, oder ein langes, aber ruhmloses, unbedeutendes Leben? Die Interpreten haben mehrheitlich dafür gestimmt, dass Achill das erstere vorziehe: kurz, aber ruhmreich. Aber Achilleus sagt an dieser Stelle im Zusammenhang gesehen ja gerade das Gegenteil! Er sagt: „Morgen segle ich nach Hause, denn das Leben ist mir mehr wert als alle Schätze. Und auch allen anderen empfehle ich das gleiche." (9, 356-361). Natürlich - das klingt nach Frustration, nach einem verbitterten, vielleicht gar schmollenden "Jetzt mag ich nicht mehr!“ Nur setzt er hinzu: „Denn das Ziel, um das es hier geht, ist unerreichbar - unerreichbar, weil Zeus seine Hand über Troia hält" (9, 417-420). Das ist keine Absage an das $\kappa \lambda \dot{\varepsilon}$ o -, aber es ist die uns schon bekannte Achilleische rationale Reflektiertheit. Achill spricht hier nach fast 10 Jahren fruchtlosen Kämpfens! Sein Leben einzusetzen für ein Ziel, von dem man weiß, dass es nicht erreichbar ist - das ist schlicht unvernünftig. Denn so wird man ja auch das wahre $\kappa \lambda \dot{\varepsilon}$ oc ä $\varphi \theta$ ı $\tau$ ov nie erreichen! Wenn der Tod sinnlos ist, dann lieber leben als sterben. Nicht um des Lebens willen leben, aus purer Lebensgier - das sicher nicht! -, aber leben, weil das Leben für alles andere, wirklich Sterbenswerte, Voraussetzung ist. Achill strebt nicht nach einem $\kappa \lambda \dot{\varepsilon} \circ \varsigma$ ä $\varphi \theta \imath \tau o v$, das durch den puren Tod erreicht wird, so sinnlos der auch ist, nicht nach dem Horazischen unbedingten dulce et decorum est pro patria mori - nicht nach dem simplen sogenannten Heldentod als solchen strebt er, sondern nach einem Heldenleben, in dem ein wahrer Heldentod der konsequente Schlusspunkt ist. Der Dichter scheint die einfache Alternative „kurzes ruhmreiches Leben“ vs. „langes ruhmloses Leben“ überwinden zu wollen. Der höchste Wert, scheint er sagen zu wollen, ist ein wirklich und wahrhaftig ruhmvolles Leben, das durch große ehrenvolle und anerkannte Taten für ein als sinnvoll erkanntes Ziel geprägt ist. Für dieses Ziel, wenn es denn sein muss, auch zu sterben - das ist wahrer Heldentod! Ein „Hurra!Tod" nicht! - Das ist nicht ganz leicht zu verstehen. Man kann lange darüber nachsinnen. 
Aber es zeigt Homers Achilleus erneut als einen Menschen, der alles andere ist als ein Schlagetot. Er macht sich Gedanken, Gedanken über die Grundfragen des Lebens. Er reflektiert. Er wägt ab. Dass dahinter der Dichter steht, der ja die Figur so zeichnet, dass also eigentlich Homer es ist, der in dieser Weise hin- und herdenkt, abwägt, zweifelt — das liegt auf der Hand.

Gerade dies aber, das Vermeiden des Schwarz-Weißen, das Problematisieren, das Homer in seinem Achilleus Gestalt werden lässt - das macht diesen Achilleus zu einem Helden, der das traditionelle Heldenbild der traditionellen Heldendichtung transzendiert. Homers Achilleus ist vermutlich für das primäre Publikum eine tiefere, eine erstaunlichere und darum um so anziehendere Verkörperung des alten Heldenideals gewesen, als alle Heldenfiguren vorangegangener Sängerdichtungen es je gewesen waren.

Und das ist es wohl, was Homers Achilleus - und damit zugleich seinem Schöpfer Homer - am Ende ein $\kappa \lambda \dot{\varepsilon} \circ \varsigma$ ă $\varphi \theta \imath \tau o v$, einen unvergänglichen Ruhm von anderer, höherer Art, eingetragen hat - ein $\kappa \lambda \varepsilon \dot{\varepsilon}$, , das die Figur Achilleus bis heute am Leben hält.

\section{Bibliographie}

Edwards M. W. Homer: Poet of the Iliad. Baltimore-London, Johns Hopkins University Press, 1987.

Effe B. Der Homerische Achilleus. Zur gesellschaftlichen Funktion eines literarischen Helden. Gymnasium 1988, 95, 1-16.

Fagles R. (Transl.) Homer. The Iliad. Intr. and Notes by B. Knox. New York, Viking, 1990.

Goetsch S. Rezension zu Shay 1994. Bryn Mawr Classical Review (BMCR) 94.03.21.

Hampe R. (Übers.) Homer. Ilias. Übersetzung, Nachwort und Register. Stuttgart, Reclam, 1979.

Latacz J. Achilleus. Wandlungen eines europäischen Heldenbildes. Stuttgart-Leipzig, Teubner, 1995; ${ }^{2} 1997$. Nachdruck in: T. Greub, K. Greub-Frącz, A. Schmitt (Hg.) Homers Ilias. Studien zu Dichter, Werk und Rezeption, Berlin-Boston, W. de Gruyter, 2014, 267-346.

Latacz J. Zur Struktur der Ilias, in: J. Latacz (Hg.) Homers Ilias. Gesamtkommentar (Basler Kommentar: BK / BKE). Prolegomena. 3. durchgesehene Aufl. Berlin-New York, De Gruyter, 2009, 145-158.

Lesky A. Homeros. Sonderdruck aus der RE Suppl. XI. Stuttgart, Druckenmüller 1967.

Schadewaldt W. (Übers.) Homer. Ilias. Frankfurt am Main, Insel, 1975.

Shay J. Achilles in Vietnam: Combat Trauma and the Undoing of Character. New York, Athenaeum, 1994.

Taplin O. Homer's use of Achilles' earlier campaigns in the Iliad, in: J.Boardman, C.E. VaphopoulouRichardson (eds). Chios. A Conference at the Homereion at Chios 1984. Oxford, Clarendon, 1986, $15-19$.

Weinrich B.H. Die fast vergessene Ehre, in: Weinrich (Hg.) Literatur für Leser. Stuttgart, W. Kohlhammer, 1971, 164-180.

Wolf Chr. Kassandra. Erzählung, Darmstadt u. Neuwied, Luchterhand 1983 u.ö. (übers. in zahlreiche Sprachen).

Voß J.-H. (Übers.) Homers Werke übersetzt. Die Ilias neu, die Odyssee umgearbeitet. 4 Bde. Bd. 1. Homers Ilias I-XII; Bd. 2. Homers Ilias XIII-XXIV. Altona, Bei I. F. Hammerich, 1793.

P. Wülfing P. Der Kassandra-Mythos und Christa Wolfs Erzählung, in: Chr. Neumeister (Hrsg.) Antike Texte in Forschung und Schule, Frankfurt am Main, Diesterweg, 1993, 301-318.

Received: 02.02.2018

Final version received: 15.04 .2018

\section{Вопросы к гомеровскому Ахиллу}

\section{Иоахим Латач}

Новейшие интерпретации Гомера при всей их метапоэтической глубине обходят стороной стержневые темы «Илиады» и главную из них - гнев Ахилла. Между тем, центральный образ поэмы, а значит, и замысел автора, понимают превратно. Ахилл оста- 
ется жертвой принятых еще в старинной филологии, получивших распространение в учебной и художественной литературе, упреков: его признают бездумно упрямым, бездумно жестоким, вообще - бездумным. Стоит, однако, задать герою четыре простых вопроса - и картина меняется. 1) «Какова твоя роль в “Илиаде”?» Ответ: «Главная!». Действительно, даже когда мы не видим его на сцене, внимание сосредоточено на нем. 2) «В чем причина твоего гнева и отказа от битвы?» Он ответит, что нанесенное при всех оскорбление провоцирует не мгновенный гнев, но въедающееся в душу возмущение несправедливостью. Почему он не принял дары? Стремление Агамемнона купить покорность героя очевидно и подчеркнуто самим поэтом. Дары были слишком щедрыми, чтобы их принять: «Мне мешает не упрямство, а самоуважение!». 3) «Почему же ты вернулся в битву?» Ахилл первенствует и в дружбе. Смерть Патрокла причиняет ему такую боль, что все, включая обиду, затмевается. Беспощадное истребление троянцев объяснимо не жестокостью и уж тем более не бездумной жестокостью. Он ищет, чем унять свою боль. 4) «В чем же для тебя высшая ценность?» Привычный ответ - слава, «невянущая слава». Сам Ахилл дает другой ответ: для него высшей ценностью является жизнь - единственное, что невозможно вернуть. Конечно, жизнь ради славы, пусть и краткая. Однако знаменитая дилемма решается отнюдь не в плакатном духе: Ахилл размышляет, стоит ли оставаться под Троей, если сам Зевс не хочет, чтобы город был взят. Говорящий чаще других героев (ему принадлежит наибольшее количество речей), главный герой «Илиады» напряженнее других старается уяснить себе смысл и значение происходящего. Нет сомнений в том, что в этой рефлексии слышен голос самого Гомера.

Ключевые слова: Ахилл, Илиада, Гомер, греческий героический эпос. 